\title{
From the Analysis of the Brain Images to the Study of Brain Networks Using Functional Connectivity and Multimodal Brain Signals
}

\author{
Fabio Babiloni
}

Received: 20 April 2010/Accepted: 22 April 2010/Published online: 8 May 2010

(C) Springer Science+Business Media, LLC 2010

This special issue is entitled "Cortical network analysis with neuroelectromagnetic and hemodynamic signals." This peer-reviewed special issue is the product of a 2-day workshop held at the University of Rome Sapienza in May 2009, during the bi-annual joint conference of two international scientific societies: The International Society for Bioelectromagnetism (ISBEM) and the International Society for Non-invasive Functional Source Imaging (NIFSI). We are grateful for the support of the European Union, through the COST Action BM0601 "NeuroMath". These two scientific societies are very active, organizing periodic conferences during which the state of the art in the area of estimation of brain activity and connectivity is presented and discussed. The next joint conference of these two societies will be held in Banff, Canada in May 2011.

\section{Methodological Studies for Improving the Estimation of the Cerebral Activity}

The special issue starts with innovative methodologies for increasing the capability of understanding the spatio-temporal characteristics of brain activity from neuroelectromagnetic measurements. De Lucia and colleagues (2010) demonstrate a methodology that compares on the same dataset of single EEG trials the brain topographies obtained by applying Independent Component Analysis (ICA) and a

F. Babiloni $(\bowtie)$

IRCCS Fondazione Santa Lucia, Rome, Italy

e-mail: fabio.babiloni@uniroma1.it

F. Babiloni

Department of Physiology and Pharmacology, University of

Rome, Rome, Italy clustering algorithm. Although these two approaches are very different conceptually and technically, the spatial distributions obtained by the two methodologies significantly correlated, demonstrating the robustness of their underlying assumptions.

The estimation of intracranial brain activity from EEG/ MEG measurements is sometimes performed with a class of inverse solutions termed "beamformers". In the second contribution of this special issue, Vrba and colleagues (2010) proposed a new beamformer that combines the Signal Space Separation and a standard beamformer (called by the Authors SSS beamformer). The authors show that the SSS beamformers are computationally more efficient than the conventional beamformers in different operative conditions, for both deep and superficial cerebral sources. In a third paper oriented to the methodological aspects, Congedo and coworkers (2010) present a study of the coherence profile (dependence) of robust eyes-closed resting EEG sources isolated by group blind source separation (gBSS). A large number of subjects are employed in this study aimed to show the organization of the extracted EEG components in two distinct networks. Within each network such components oscillate coherently with multiple-frequency dynamics, whereas between networks they exchange information at non-random multiple time-lag rates.

\section{Multimodal Studies for Improving the Estimation of Cerebral Activity}

Beside the use of novel methodologies for improving the estimation of cortical activity, this special issue includes a series of papers that illustrate the advantage of the use of multimodal information ranging from the estimation of 
cerebral activity, as detected by hemodynamic and neuroelectromagnetic signals, to magnetoencephalographic (MEG) and electrocorticographic (EcoG) signals. In addition, variation in cardiac rate and skin impedance were used as signs of variation of autonomic activity. The general conclusion of these papers is that such a multimodal approach is useful for gaining additional information about the "brain state" that otherwise would be not gained by using the data from a single modality alone. In fact, it is largely believed that the use of different information from neuroelectromagnetic and hemodynamic modalities can improve the quality of cerebral source reconstructions. In this context, it is important (1) to model the dependency between the electromagnetic and the hemodynamic signals and (2) to generate methods that take into account such inter-dependency and combine the results from the different modalities in a unique framework.

In this special issue two papers attempted to clarify such issues. Bojak and colleagues (2010) demonstrated that a mean field model of brain activity can simultaneously predict EEG and fMRI BOLD with realistic signal expression. These authors provided an application using anatomical geometry based on structural MRI and including long-range connectivity between brain regions. The integration of a mean field model with realistic geometry data is indeed an important first step towards the modelbased integration of multimodal neuroimaging.

Mantini and coworkers (2010) analyzed the use spatial and temporal ICA on the information gathered from hemodynamic and neuroelectrical modalities. In this study, the event-related potentials in response to visual target detection were first recorded and then subjected to temporal ICA. The resulting components were then localized with the weighted minimum norm algorithm using the taskrelated fMRI networks (estimated with the spatial ICA) as priors. Results confirm that two different components, commonly observed in EEG when presenting novel and salient stimuli respectively, are related to the neuronal activation in large-scale networks, operating at different latencies and associated with different functional processes.

The use of multimodal data is also crucial in a clinical context. Huiskamp and coworkers (2010) demonstrate the validity of the comparative analysis of magnetoencephalographic (MEG) and electrocorticographic (EcoG) data. In fact, in their paper the authors investigated the relationships between the estimated sources of MEG spikes and the location, distribution and size of interictal spikes in the invasive ECoG of a group of 38 epilepsy patients, who were being monitored for pre-surgical evaluation. The conclusion of this paper is that all MEG spikes are associated with an ECoG spike that is among the three highest ranked in a patient.
As a further example of the multimodal integration of data, Vecchiato et al. (2010) present the use of different data from the central and autonomic nervous systems in relation to the assessment of cognitive and emotional activities during the observation of TV commercials. Such assessment was done by using EEG, heart rate, and galvanic skin responses. The authors show how cerebral activity during the observation of TV spots that are subsequently remembered by the subjects are associated with an increased power spectral activity in the theta band in left prefrontal areas. In addition, it was observed that the emotional activity related to the TV commercials that subjects liked was associated with increased heart rate.

Finally, Bianchi and coworkers (2010) investigated which evoked response component occurring in the first $800 \mathrm{~m} \mathrm{~s}$ after stimulus presentation was most suitable to be used in a classical P300-based Brain-Computer Interface (BCI) speller protocol. This study was performed by using both MEG and EEG data in different subjects. The main conclusion of this work was that early occipital components $(0-250 \mathrm{~m} \mathrm{~s})$, present in both EEG and MEG modalities, could be useful to improve the automatic classification accuracy for this class of BCI device.

\section{Application of EEG Analysis and Source Localization}

This special issue also includes two interrelated papers in the area of Brain Computer Interface by Vidaurre and Blankertz (2010) and by Sanelli et al. (2010). In Sannelli et al., the problem of the selection of the optimal placement of the electrodes for a BCI system is addressed. In fact, there is a typical trade-off in BCI systems. While a setup with few channels is more convenient and requires less preparation time, a dense placement of electrodes provides more detailed information and henceforth could lead to a better classification performance. In their paper Sanelli et al. used a Common Spatial Pattern (CSP) algorithm in order to classify the sensorimotor rhythms detected in a large population of subjects. They compared different configuration of channels with a gold standard obtained by covering the scalp of the subjects with 119 electrodes. The results they obtained in 80 participants are that when only a few trials are available a configuration with 22 electrodes returns good results in classification of such EEG rhythms. In the second paper related to the BCI field, Vidaurre et al. addressed the issue of how to improve $\mathrm{BCI}$ performance in users that for one reason or another are not able to properly control a BCI system (called BCI illiteracy). BCI illiterates, according to the authors, typically range from 15 to $30 \%$ of the investigated population. The authors hypothesized that such a population could be trained using a more sophisticated classifier along with 
machine learning techniques. This approach allowed users who were previously unable to achieve successful feedback to gain significant control over the BCI system during the successive phase of the training session.

The application of the state of the art techniques in cerebral source localization by using neuroelectrical modalities is presented in a paper by Koles et al. (2010) that presents a quantitative EEG study of gender-related differences in brain function. The study involved estimates of both the source-current power density in the brain and the complex coherence between different regions in the brain. Gender effects are shown in terms of differences in both lateralized source power and complex coherence in response to verbal and spatial cognitive challenges. In agreement with neuroimaging findings, their results showed gender differences between cortical activity in males and females during spatial and verbal tasks.

\section{The Use of Functional Connectivity as a Tool for Assessing Brain Functions in Scientific and Clinical Contexts}

A particular importance in the workshop was given to the issue of the estimation of functional connectivity. This hot theme in neuroscience is illustrated by a series of papers that use the concept of functional connectivity in different tasks in healthy populations as well as patients. The paper of Blinowska and colleagues (2010) employs a timevarying estimator of functional connectivity, named Shorttime Directed Transfer Function (SDTF). SDTF is an estimator based on a multivariate autoregressive model that determines the propagation as a function of time and frequency. Such an estimator is employed for studying the time-varying connectivity patterns in a group of nine subjects during a cognitive task, overcoming the assumption of stationarity of the EEG data that it is assumed by the standard application of functional connectivity methods.

The application of the estimation of cortical connectivity in a clinical context is presented in the study of De Vico Fallani and coworkers (2010), where they studied the structural changes of the brain functional network in a group of schizophrenic patients during a 2-back working memory task. In particular, the authors show that in the Alpha2 frequency band $(11-13 \mathrm{~Hz})$ the cortical functional networks of the schizophrenic patients present the largest differences when compared with those of a group of control subjects.

The paper by Pollonini and coworkers (2010) employs spectral coherence and Granger causality (another connectivity estimator) to separate patients in minimally conscious state (MCS) from patients with severe neurocognitive disorders (SND) that show signs of awareness. The study shows that different patterns of functional connectivity estimated by Granger causality could be employed for classifying subjects affected by severe traumatic brain injury.

The goal of this special issue of Brain Topography is to convey several main messages of this intense mini-conference. The topic of the conference focused on the marriage between methods and applications in the field of modern brain imaging techniques. The methods and the data presented in such a conference are moving along the relatively new road that starts with brain topography, passes through the use of brain images, and culminates with the estimation of brain functions by employing concepts related to brain networks. Such a path is still to be fully performed in neuroscience, but this special issue would like to bring to the attention of the readership of the journal several papers that are moving in this direction.

\section{References}

Bianchi L, Sami S, Hillebrand A, Fawcett IP, Quitadamo LR, Seri S (2010) Which physiological components are more suitable for visual ERP based brain-computer interface? A preliminary MEG/EEG study. Brain Topogr 23(2). doi:10.1007/s10548-0100143-0

Blinowska K, Kus R, Kaminski M, Janiszewska J (2010) Transmission of brain activity during cognitive task. Brain Topogr 23(2). doi:10.1007/s10548-010-0137-y

Bojak I, Oostendorp TF, Reid AT, Kötter R (2010) Connecting mean field models of neural activity to EEG and fMRI data. Brain Topogr 23(2). doi:10.1007/s10548-010-0140-3

Congedo M, John RE, De Ridder D, Prichep L, Isenhart R (2010) On the "dependence" of "independent" group EEG sources; an EEG study on two large databases. Brain Topogr 23(2). doi:10.1007/s10548-009-0113-6

De Lucia M, Michel CM, Murray MM (2010) Comparing ICA-based and single-trial topographic ERP analyses. Brain Topogr 23(2). doi:10.1007/s10548-010-0145-y

De Vico Fallani F, Maglione A, Babiloni F, Mattia D, Astolfi L, Vecchiato G, De Rinaldis A, Salinari S, Pachou E, Micheloyannis S (2010) Cortical network analysis in patients affected by schizophrenia. Brain Topogr 23(2). doi:10.1007/s10548-0100133-2

Huiskamp G, Agirre-Arrizubieta Z, Leijten F (2010) Regional differences in the sensitivity of MEG for interictal spikes in epilepsy. Brain Topogr 23(2). doi:10.1007/s10548-010-0134-1

Koles ZJ, Lind JC, Flor-Henry P (2010) Gender differences in brain functional organization during verbal and spatial cognitive challenges. Brain Topogr 23(2). doi:10.1007/s10548-0090119-0

Mantini D, Marzetti L, Corbetta M, Romani GL, Del Gratta C (2010) Multimodal integration of fMRI and EEG data for high spatial and temporal resolution analysis of brain networks. Brain Topogr 23(2). doi:10.1007/s10548-009-0132-3

Pollonini L, Pophale S, Situ N, Wu M-H, Frye RE, Leon-Carrion J, Zouridakis G (2010) Information communication networks in severe traumatic brain injury. Brain Topogr 23(2). doi:10.1007/ s10548-010-0139-9 
Sannelli C, Dickhaus T, Halder S, Hammer E-M, Müller K-R, Blankertz B (2010) On optimal channel configurations for SMRbased brain-computer interfaces. Brain Topogr 23(2). doi:10.1007/s10548-010-0135-0

Vecchiato G, Astolfi L, De Vico Fallani F, Cincotti F, Mattia D, Salinari S, Soranzo R, Babiloni F (2010) Changes in brain activity during the observation of TV commercials by using
EEG, GSR and HR measurements. Brain Topogr 23(2). doi:10.1007/s10548-009-0127-0

Vidaurre C, Blankertz B (2010) Towards a cure for BCI illiteracy. Brain Topogr 23(2). doi:10.1007/s10548-009-0121-6

Vrba J, Taulu S, Nenonen J, Ahonen A (2010) Signal space separation beamformer. Brain Topogr 23(2). doi:10.1007/s10548-0090120-7 